\title{
Evolution of the microstructure of cobalt during diffusionless transformation cycles
}

\author{
A. Munier, J.E. Bidaux, and R. Schaller \\ Institut de Génie Atomique, Ecole Polytechnique Fédérale de Lausanne, CH-1015 Lausanne, \\ Switzerland \\ C. Esnouf \\ Groupe d'Etudes de Métallurgie Physique et de Physique des Matériaux, Bât. 502, I.N.S.A. de Lyon, \\ F-69621 Villeurbanne, France
}

(Received 29 August 1989; accepted 6 December 1989)

\begin{abstract}
Differential scanning calorimetry and transmission electron microscopy have been used to study thermal fatigue due to diffusionless phase transformation cycling in pure cobalt. Thermal cycling through the allotropic (hcp $\leftrightarrow \mathrm{fcc}$ ) transformation results in a temperature shift of the calorimetric peaks, which means a delay of the transformation. In addition, the transformation enthalpy, which is greater on heating than on cooling, diminishes when the number of transformation cycles increases. This is interpreted as being due to an evolution of the microstructure. Transmission electron microscopy shows the appearance of transformation-induced defects, which are mainly sessile dislocations. We can interpret the calorimetry results (enthalpy evolution and transformation delay) as due to the interactions between interface dislocations and these sessile dislocations.
\end{abstract}

\section{INTRODUCTION}

The stability of shape memory behavior in alloys which exhibit a thermoelastic martensitic transformation is very important for technical applications. ${ }^{1}$ The shape memory effect is directly related to the microstructure of the metal. It can then be affected by any evolution of this microstructure during aging or during thermal cycling. In order to achieve a better understanding of the interactions between microstructure and martensitic transformation, we have performed studies on the evolution of the microstructure of cobalt during thermal cycling through the transformation.

Cobalt exhibits a diffusionless phase transformation quite similar to the so-called thermoelastic martensitic transformation. The advantage of using a pure metal is that one can study thermal fatigue which is due only to an evolution of the microstructure, without perturbations by precipitation.

The allotropic phase transformation of cobalt takes place at around $420^{\circ} \mathrm{C}$ where the hcp phase (martensite), stable at low temperature, transforms to the fcc phase (austenite), stable at high temperature. This transformation is a first order transition characterized by a hysteresis of about $40^{\circ} \mathrm{C}$ and a transformation enthalpy.

Results obtained by Adams and Altstetter in pure cobalt ${ }^{2}$ show that the enthalpy variation $(\Delta H)$ during the transformation is surprisingly greater on heating than on cooling. That could be explained by the appearance of transformation-induced defects remaining in the structure. In addition, the transformation enthalpy depends on the previous thermal treatments, i.e., on the microstructure of the specimen.
Several models have been developed which show the importance of the influence of structural defects on the transformation:

(1) growth of pre-existent stacking faults $\left(\mathrm{Lysak}^{3}\right)$;

(2) relaxation of local stress due to the crossing of different stacking faults $\left(\right.$ Bollman $\left.^{3}\right)$;

(3) rotation of a Schockley partial dislocation around a dislocation with a screw component of $2 a / 3\langle 111\rangle$ $\left(\right.$ Seeger $\left.^{3,4}\right)$;

(4) reflection of partial dislocations on the surface or on the grain boundaries $\left(\mathrm{Christian}^{3}\right)$;

(5) emission of partial dislocations from grain boundaries (Kennedy et al..$^{5}$ );

(6) spontaneous nucleation of partial dislocations (Adams and Altstetter ${ }^{2}$ );

(7) occurrence of six consecutive plane blocks of the new phase, having low coherency stresses with regard to the matrix (Blaschko et al. ${ }^{6}$ ).

To summarize, each author assigns the transformation to a shear in the close-packed planes which could be due to the motion of partial dislocations across alternate close-packed planes. Consequently, the transformation depends strongly on the mobility of these partial dislocations.

The stacking faults and the dislocations in the cobalt hcp structure have been studied by transmission electron microscopy (TEM). ${ }^{7-9}$ Two kinds of stacking faults have been observed in the stacking sequence of the basal planes: intrinsic ones $\left(I_{1}\right.$ and $\left.I_{2}\right)$ and extrinsic ones $(E)$. The Burgers vectors of the partials surrounding them are of the type $\mathbf{p}+\mathbf{c} / 2=1 / 6\langle 2 \overline{2} 03\rangle, \mathbf{p}=1 / 3\langle 1 \overline{1} 00\rangle$, and $\mathbf{c} / 2=1 / 2\langle 0001\rangle$ in the case of $I_{1}, I_{2}$, and $E$, respec- 
tively. The density of $I_{1}$ faults is about two orders of magnitude larger than the density of $E$ faults. Only the $I_{2}$ faults can be produced by shear in the basal plane.

The partial dislocations are the ones which surround the stacking faults. The perfect dislocations can have the following Burgers vectors: $\mathbf{a}=1 / 3\langle 11 \overline{2} 0\rangle, \mathbf{c}=$ $\langle 0001\rangle$, and $\mathbf{c}+\mathbf{a}=1 / 3\langle 11 \overline{2} 3\rangle$. Except for a few short segments of type $\mathbf{c}$, all these dislocations are observed in the basal planes. They are mainly partial dislocations of type p ("grown-in" dislocations) arranged in bundles and networks. The networks also contain several straight dislocations, which lie along $\langle 11 \overline{2} 0\rangle$, and are sessile partial dislocations of type $\mathbf{p}+\mathbf{c} / 2$.

The purpose of this study is to measure the thermodynamic parameters of the cobalt allotropic transformation and to follow their evolution during transformation cycling. This evolution, measured by calorimetry, is then correlated with the evolution of the microstructure, observed by transmission electron microscopy.

\section{EXPERIMENTAL PROCEDURE}

The cobalt used was a polycrystalline specimen provided by Johnson-Matthey. The nominal purity of the material was $99.99 \%$ and the average grain diameter was about $0.1 \mathrm{~mm}$. Cylindrical specimens (diameter $=3 \mathrm{~mm}$, thickness $=1 \mathrm{~mm}$ ) cut with a diamond saw were vacuum-annealed for $2 \mathrm{~h}$ at $1100{ }^{\circ} \mathrm{C}$. Transformation cycles were then performed between 200 and $530{ }^{\circ} \mathrm{C}$ in a dual elliptical radiant heater $\left(d T / d t= \pm 28^{\circ} / \mathrm{min}\right.$, helium pressure of $\left.50 \mathrm{mbar}\right)$ for the TEM samples and directly in the calorimeter for the others. Calorimetric measurements were carried out in a Perkin-Elmer Differential Scanning Calorimeter DSC-4. A reference specimen (Al) was heated or cooled from an initial to a final temperature with $d T / d t=$ $\pm 10^{\circ} / \mathrm{min}$. The power $(d H / d t)$ required to maintain the cobalt sample at the same temperature as the reference specimen was continuously measured and recorded.

The microstructure of the hcp phase has been studied by TEM. The samples were the same as the ones used for the calorimetry measurements. They were reduced to a thickness of $120 \mu \mathrm{m}$ with a wire saw (type WS $20 \mathrm{~A}$ ), and the thin foils were prepared by electrolytic polishing $\left(77 \% \mathrm{CH}_{3} \mathrm{COOH}\right.$ and $\left.23 \% \mathrm{HClO}_{4}\right)$. They were examined at $200 \mathrm{kV}$ in a Hitachi $\mathrm{H}-700 \mathrm{H}$. The TEM observations have been carried out at room temperature before any transformation and after 24 and 100 cycles of transformation.

\section{EXPERIMENTAL RESULTS}

\section{A. Calorimetry measurements}

The calorimetry results present an endothermic peak on heating (hcp $\rightarrow$ fcc transformation) and an exothermic one on cooling (fcc $\rightarrow$ hcp transformation) (Fig. 1).

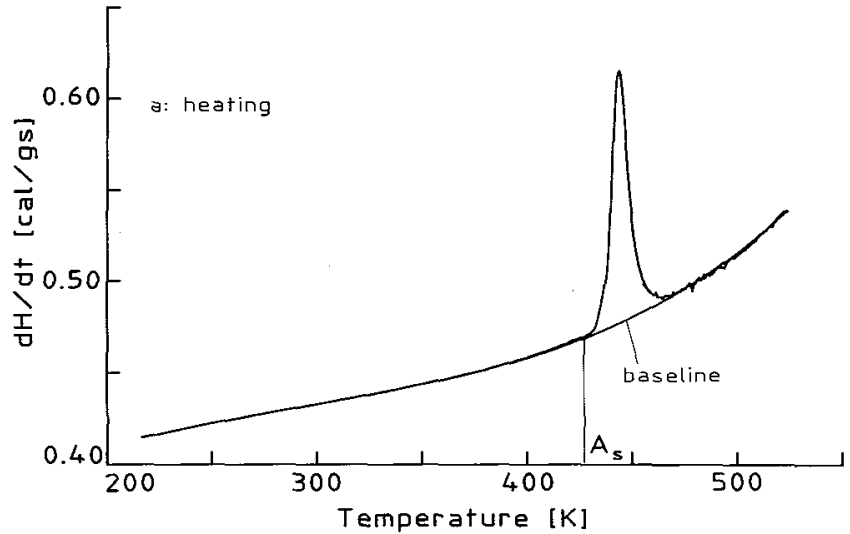

(a)

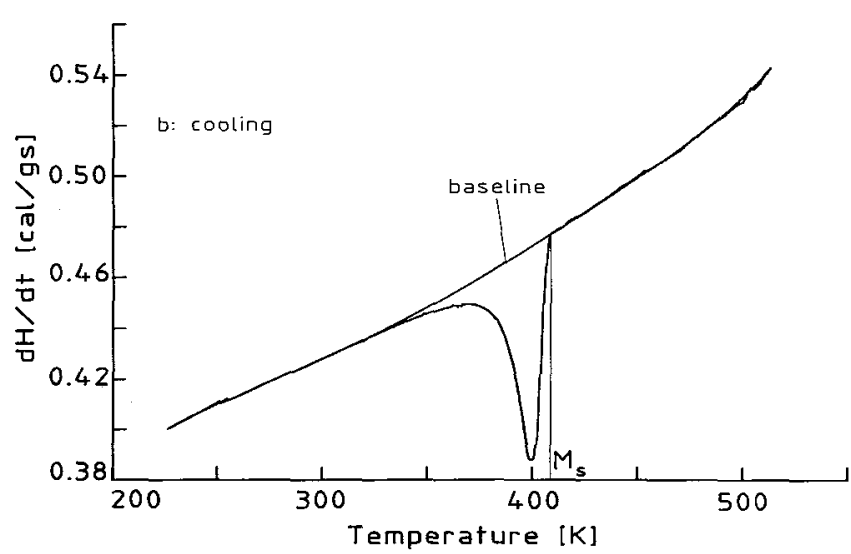

(b)

FIG. 1. Differential scanning calorimetry thermograms (first cycle): (a) on heating, endothermic hcp $\rightarrow$ fcc transformation; (b) on cooling, exothermic fcc $\rightarrow$ hcp transformation.

Different parameters can be gotten from these rough results: $A_{s}$ (Austenite start): temperature at the beginning of the transformation on heating. $M_{s}$ (Martensite start): temperature at the beginning of the transformation on cooling. $\Delta H^{h}$ : Enthalpy change during the hcp $\rightarrow$ fcc transformation. $\Delta H^{c}$ : Enthalpy change during the fcc $\rightarrow$ hcp transformation.

The hysteresis, classical for a first order transition, between the transformation on heating and on cooling is defined as $A_{s}-M_{s}$. In order to measure the enthalpy of transformation, a background must be subtracted. We should get the baseline as the result if there were no transformation. It is obtained by fitting (method of Chebyscheff) the part of the thermogram which does not depend on the transformation and by extrapolating the resultant curve over the transformation temperature range. $A_{s}$ and $M_{s}$ are the temperatures at which the signal deviates from the baseline.

The value of the transformation enthalpy $(\Delta H)$ is usually associated with the area of the transformation peak. ${ }^{11}$ In this study, the accuracy of this method is 
faulty for two main reasons: the end of transformation is not well defined on the peak, and corrections taking into account the hysteresis width and the nonlinearity of the specific heat would have to be calculated. Therefore an "integration method" is used. $\Delta H^{h}$ and $\Delta H^{c}$ are not explicitly resolved. The calculation of $\int_{T_{0}}^{T}(d H / d t) d T$ allows the determination of the enthalpy variation between the temperature $T_{0}$, situated about $10^{\circ}$ before the transformation $\left(T_{0}=425^{\circ} \mathrm{C}\right.$ on heating and $415{ }^{\circ} \mathrm{C}$ on cooling), and the temperature $T$. Figure 2 , which shows the results obtained by this method, gives a first idea of the evolution of $\Delta H$ with the transformation cycles.

For each measurement, we calculate the enthalpy change in the following way:

on heating: $\Delta H^{h}=\int_{425}^{460} \frac{d H}{d t} d T$
on cooling: $\Delta H^{c}=\int_{415}^{380} \frac{d H}{d t} d T$

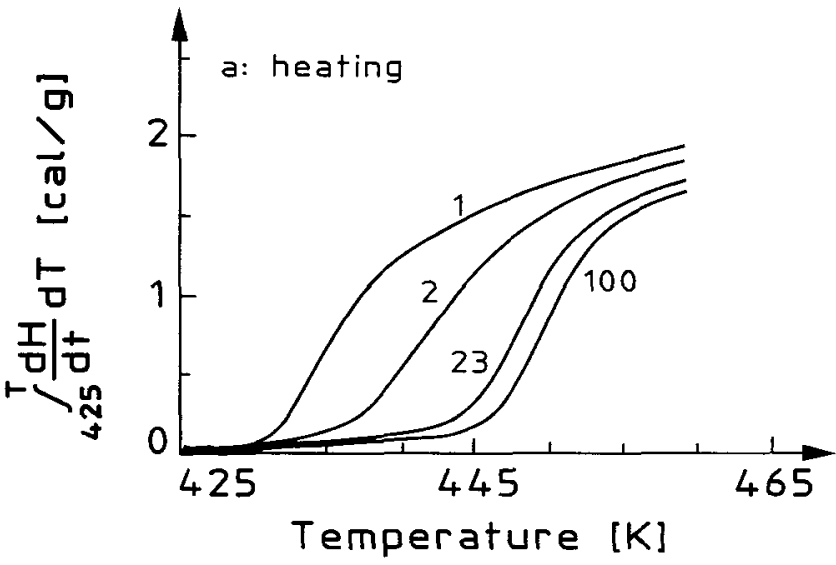

(a)

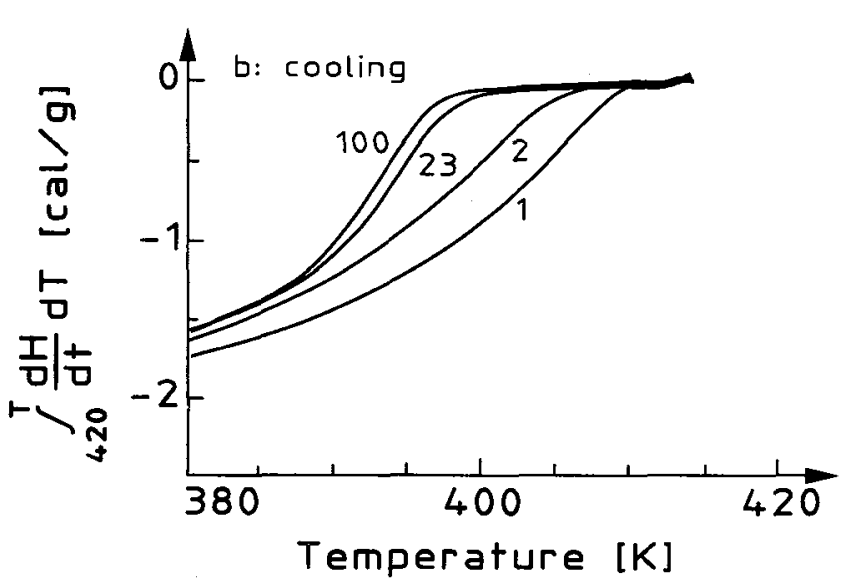

(b)

FIG. 2. Variation of enthalpy during transformation on heating (a) and on cooling (b) for different cycles $(1,2,23$, and 100).
These values are plotted in Fig. 3. For each cycle, the enthalpy change is greater on heating than on cooling $\left(\Delta H^{h}>\Delta H^{c}\right)$. This agrees with the results of Adams and Altstetter. ${ }^{2}$

In addition, the systematic investigations performed in the present research show clearly that the values of $\Delta H^{h}$ and $\Delta H^{c}$ diminish when the number of transformation cycles increases. They reach equilibrium values separated by about $0.08 \mathrm{cal} / \mathrm{g}$. The hysteresis width $\left(A_{s}-M_{s}\right)$ increases with the number of transformation cycles $\left(A_{s}\right.$ increases and $M_{s}$ decreases, Fig. 4). Once again, these two temperatures reach equilibrium values:

$$
A_{s}^{\text {eq }}=446^{\circ} \mathrm{C} \text { and } M_{s}^{\text {eq }}=399^{\circ} \mathrm{C} \text {. }
$$

The evolution of $\Delta H^{h}, \Delta H^{c}, A_{s}$, and $M_{s}$ is considerable during the first transformation cycles (up to the twentieth), and less significant during the following ones. This is the reason why the TEM observations of the microstructure have been carried out before any transformation and after 24 and 100 transformation cycles.

\section{B. TEM observations}

Figure 5 shows the microstructure of cobalt at room temperature after a few transformation cycles. Selectedarea diffraction patterns have revealed that all the specimens present an hop structure. There is no retained fcc phase.

The boundaries between the different hep zones are parallel to well-defined crystallographic planes: basal planes of one or the other adjacent area (they appear parallel to dislocations or to stacking faults). These basal planes are related to the close-packed planes $(\{111\})$ of the fcc structure. Thus, if the different hcp zones proceed from the same fcc area, their basal planes are inclined at an angle of $71^{\circ}$. Effectively, in some cases, such an angle of $71^{\circ}$ can be measured

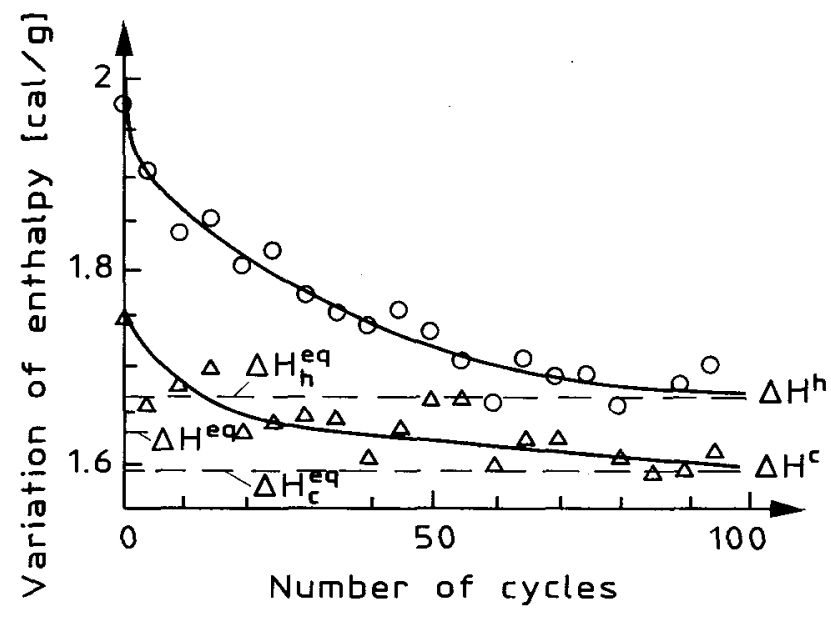

FIG. 3. Effect of cycling on the enthalpy change: between 425 and $465^{\circ} \mathrm{C}$ on heating $\left(\Delta H^{h}\right.$ : open circles) and between 415 and $380^{\circ} \mathrm{C}$ on cooling ( $\Delta H^{h}$ : triangles). 


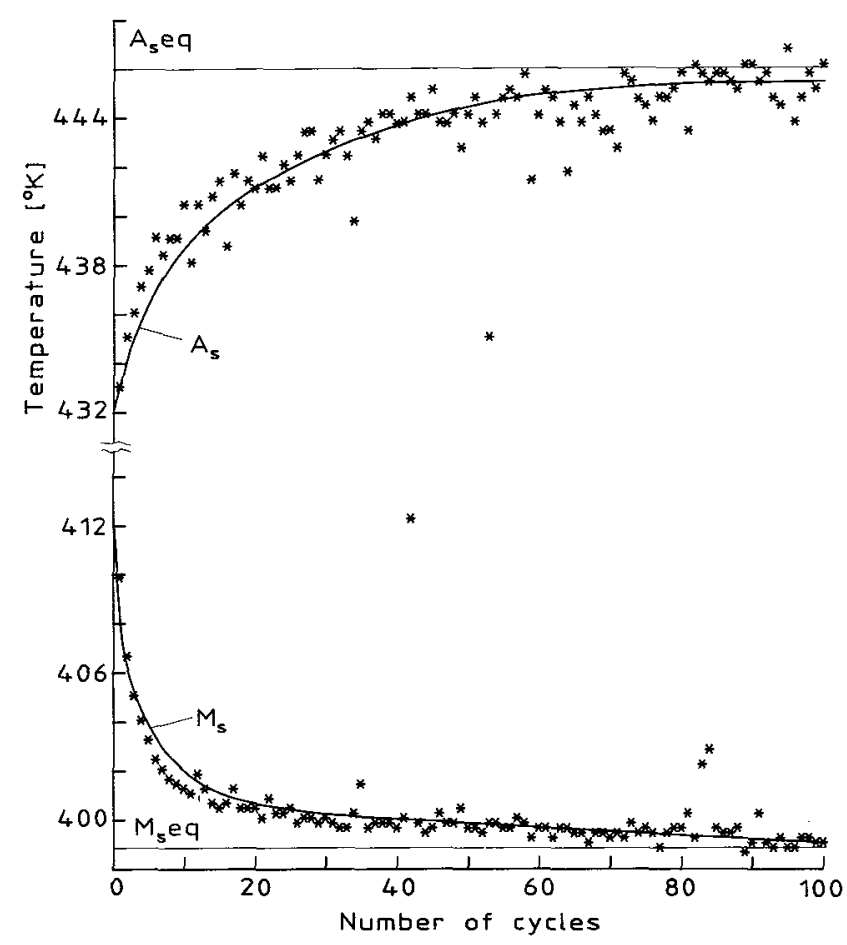

FIG. 4. Effect of cycling on $A_{s}$ and $M_{s}$ : increase of the hysteresis width.

among the different variants of the hep phase. But sometimes this angle is undefined because the various hcp zones proceed from differently orientated fcc areas.

Figures 6(a), 6(b), and 6(c) show one of these hcp variants. The basal planes are in the Bragg position $(\mathbf{g}=[0002])$. With such an orientation, the stacking faults are out of contrast, when most of the dislocations appear [only dislocations with Burgers vectors in the basal plane (a or p) are out of contrast]. Except for a few short segments (Fig. 7), all the dislocations are in the basal planes. Observing the same zones with other orientations has revealed that there are only a few p-type dislocations in the structure, so we will consider only the dislocations which are in contrast with $\mathbf{g}=[0002]$. The greatest part of them are $\mathbf{p}+\mathbf{c} / 2$-type partial dislocations, sessiles, lying along $\langle 11 \overline{2} 0\rangle$.

There is an evolution of the microstructure when the sample is cycled around the transformation [Figs. 6(a), 6(b), and 6(c)]. Before any transformation [Fig. 6(a)], there are only a few of these $\mathbf{p}+\mathbf{c} / 2$ dislocations. After 24 transformation cycles [Fig. 6(b)], their density is obviously higher and the structure is sprinkled with dislocation loops. Between 24 and 100 transformation cycles [Fig. 6(c)], the evolution is less significant.

\section{DISCUSSION}

Our calorimetry measurements provide some interesting results. The increase of the hysteresis $\left(A_{s}-M_{s}\right)$ with the transformation cycles indicates that the nucleation of the new phase is always more difficult. This could be explained by an increase of the lattice defects density. These defects could interact with the transformation nucleating and propagating dislocations. In this way, the excess of enthalpy measured upon heating $\left(\Delta H^{h}-\Delta H^{\text {eq }}\right.$, Fig. 3) corresponds to the amount of energy needed for the formation of transformationinduced defects on heating. After each transformation, the need of these defects would decrease. On cooling, the sample hands back only a part of the energy it has accumulated on heating. The remaining part of this energy $\left(\Delta H^{h}-\Delta H^{c}\right)$ has been used for the creation of these defects which remain in the structure after the transformation cycle. These assumptions are corroborated by the TEM observations.

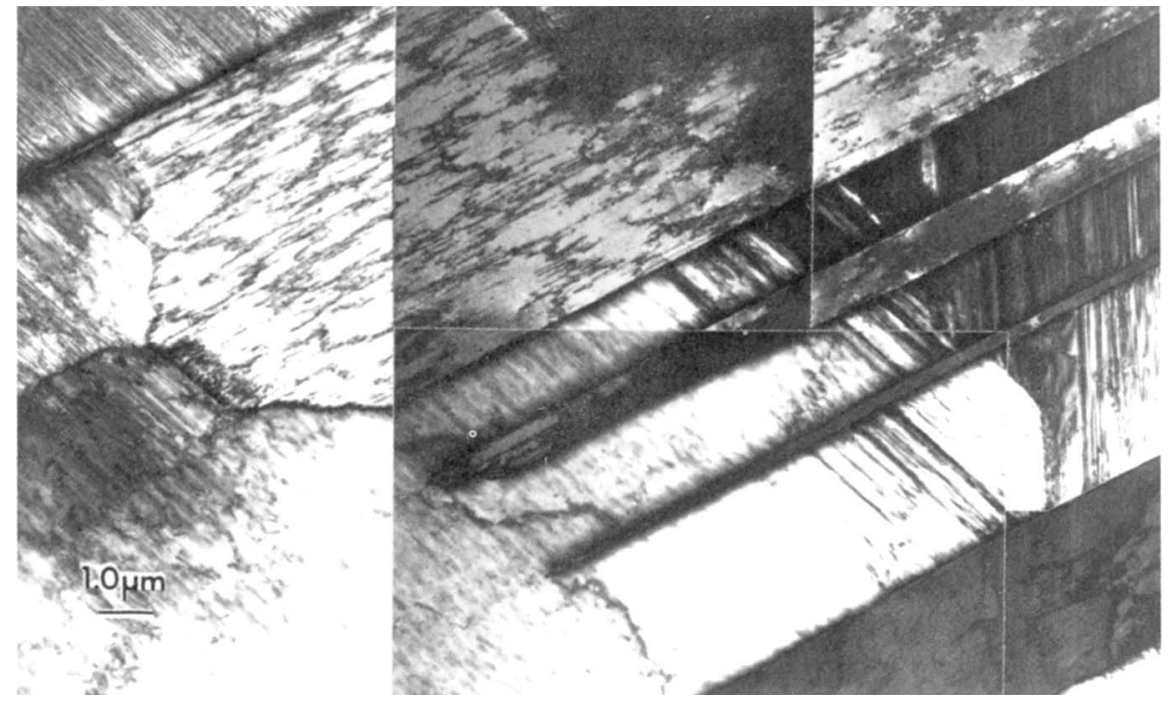

FIG. 5. Large region of already transformed cobalt at room temperature; each area has hep structure. 
The persistent transformation-induced defects are mainly the $\mathbf{p}+\mathbf{c} / 2$-type partial dislocations lying along $\langle 11 \overline{2} 0\rangle$. Most of them have been effectively created during the first transformation cycles. In addition to these sessile dislocations, dislocation loops have also been created, even if the transformation enthalpies have reached their equilibrium values. The creation of these loops needs some energy upon heating and upon cooling. We ask, what can explain the difference between $\Delta H_{h}^{\text {eq }}$ and $\Delta H_{c}^{\text {eq }}$ ?
The appearance of these $\mathbf{p}+\mathbf{c} / 2$ dislocations and of these dislocation loops can be explained as follows. The observed dislocations are particular: they are partial, $\mathbf{p}+\mathbf{c} / 2$-type, sessile, and they border $I_{1}$ stacking faults in the basal planes. On the other hand, the transformation shear does not modify the Burgers vectors and these dislocations are glide dislocations in the fcc structure $(\mathbf{b}=a / 2\langle 110\rangle)$. Their glide planes are the close-packed planes which are not parallel to the transformation planes. During the hcp $\rightarrow$ fcc transforma-

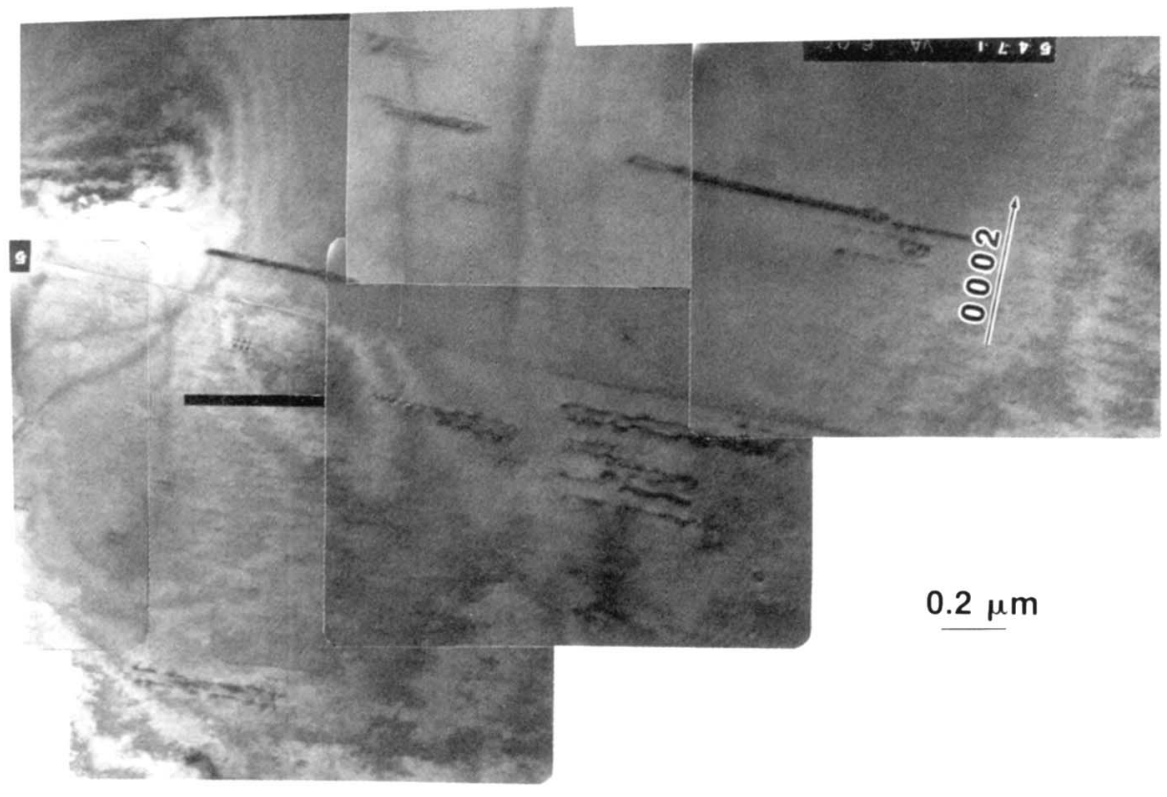

(a)

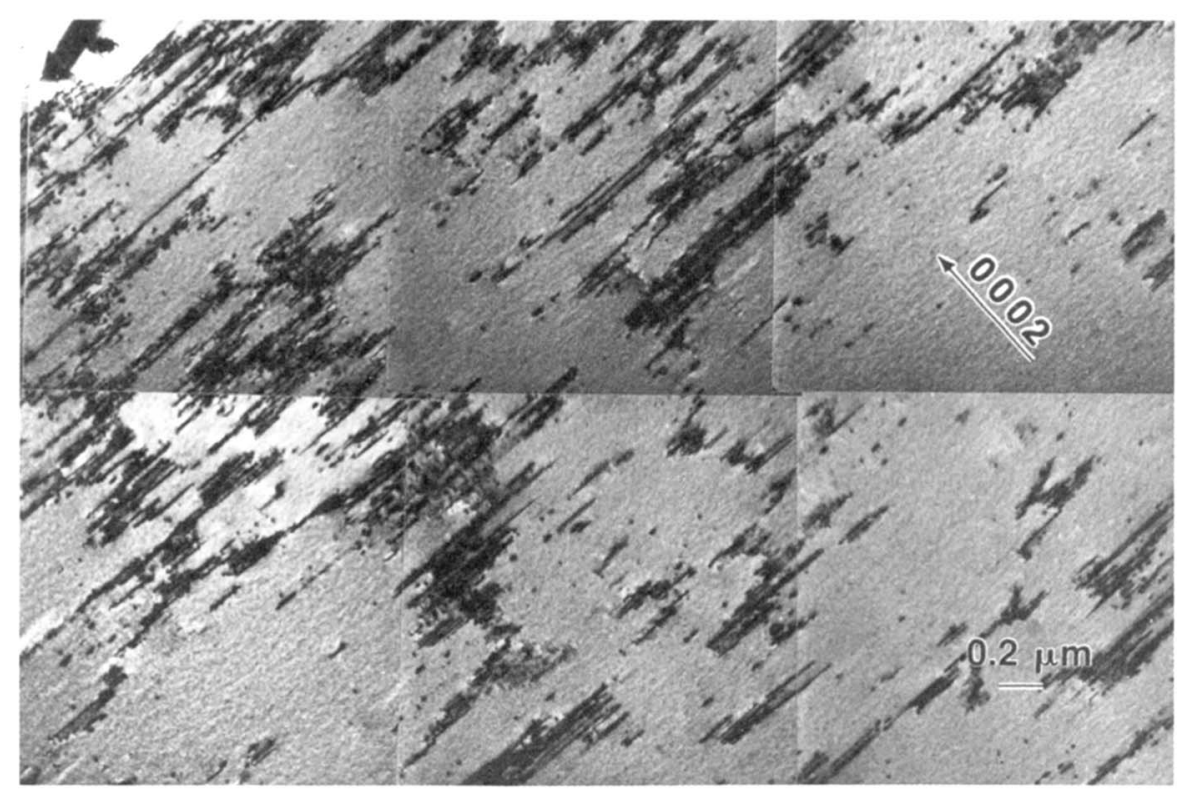

(b)
FIG. 6. Effect of cycling on the microstructure of one of the hcp areas: (a) before any transformation, (b) after 24 transformation cycles, and (c) after 100 transformation cycles, $(\mathrm{g}=[0002])$. 


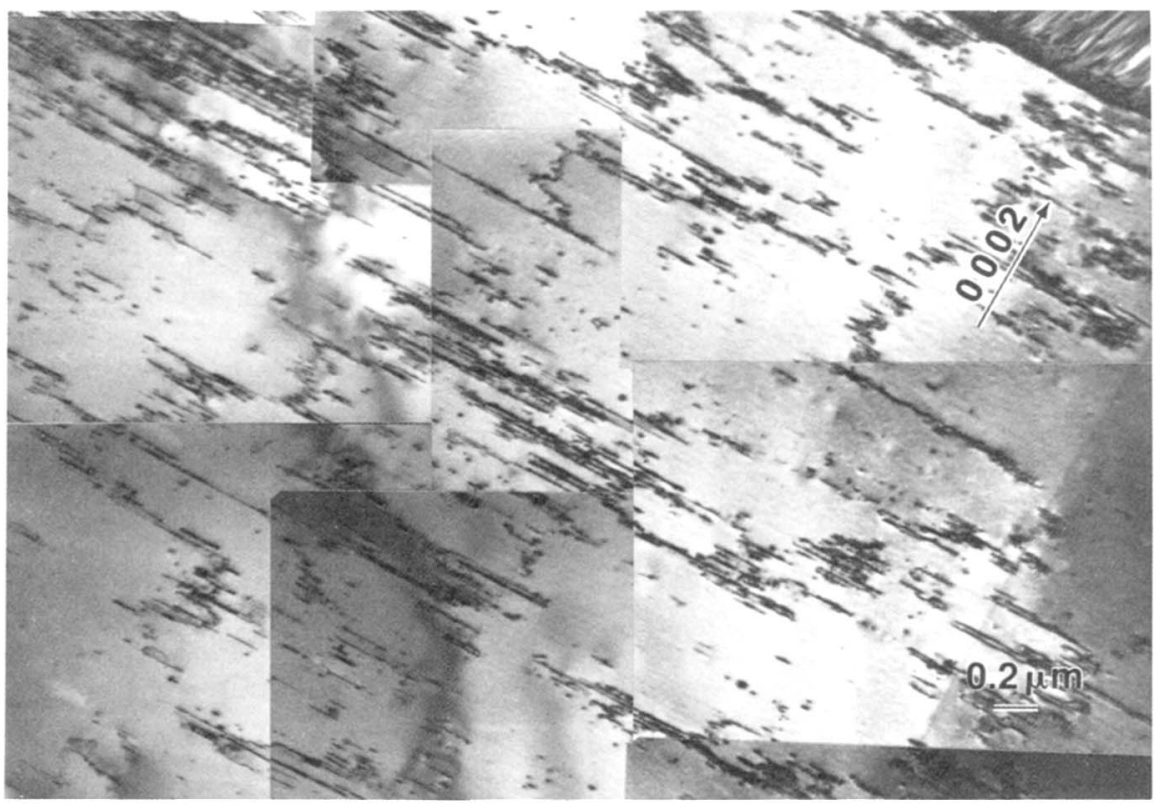

FIG. 6. (continued)

(c)

tion, the new phase is nucleated in the hcp matrix. It is associated with an expansion of $0.042 \%$ normal to the basal plane and a contraction of $0.03 \%$ in the basal plane. ${ }^{2}$ So, each nucleus has to be adjusted to the others and to the matrix, especially in polycrystalline cobalt where incompatibilities of strain exist at grain boundaries.

This adaptation is performed with the creation of perfect dislocations, which is much easier in the fcc structure (12 glide systems) than in the hcp structure (3 glide systems). So the Burgers vectors of these adaptation dislocations are $a / 2\langle 110\rangle$. They are either on the transformation planes or on the other fcc close-packed planes. During the following fcc $\rightarrow$ hcp transition, the ones which lie on the transformation planes dissociate and allow the nucleation and the propagation of the hcp phase. On the other hand, the ones which lie on the other $\{111\}$ planes cannot dissociate or stay in the same places for energetic reasons (it is too difficult to have a stacking fault in an hcp plane other than the basal one). So the transformation front pushes them away in their glide planes up to grain boundaries or to the surface of the sample, giving rise to slip bands, which have been observed by Bibring $e t a l{ }^{10}$ But if the

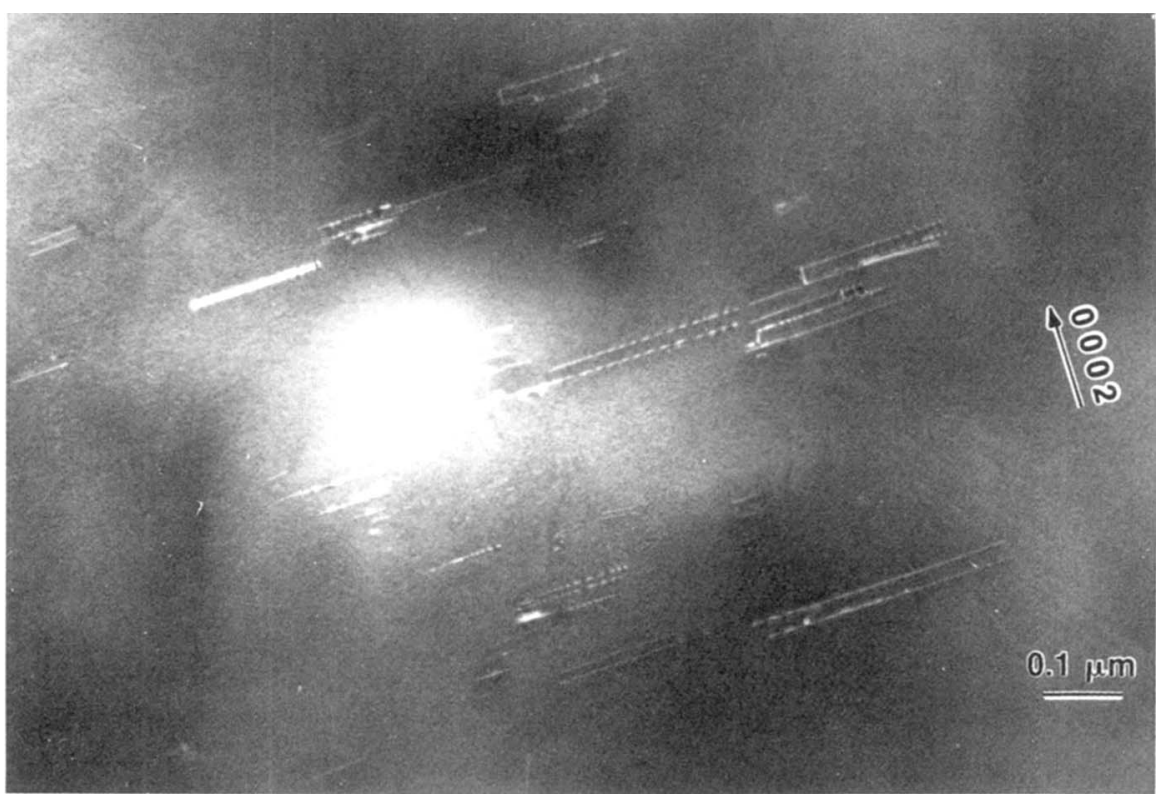

FIG. 7. Dislocation structure: except short segments (c type), every dislocation is in the basal plane (weak beam $\mathbf{g} / \mathbf{3 g}, \mathbf{g}=[0002])$. 
dislocations are pinned, they cannot be pushed away; they can only be laid in the transformation planes, in the $\langle 11 \overline{2} 0\rangle$ directions, as observed by TEM.

During the following hcp $\rightarrow$ fcc transformation, the adaptation dislocations which have been pushed away have to be replaced, while the pinned dislocations simply get back into their adaptation positions. This explains the diminution of $\Delta H$ during the transformation cycles. The partial dislocations which participate in the nucleation and propagation of the new phase are impeded by the forest of these sessile adaptation dislocations, so that $A_{s}$ increases and $M_{s}$ decreases. On the other hand, jogs can appear on the mobile dislocations, due to this interaction. They are dragged, producing a lot of vacancies which precipitate and form dislocation loops. These loops are created on heating and on cooling and they remain in the structure. This mechanism needs some energy, which explains the difference of $0.08 \mathrm{cal} / \mathrm{g}$ measured between $\Delta H_{h}{ }^{\text {eq }}$ and $\Delta H_{c}{ }^{\text {eq }}$.

Qualitatively, this model allows an explanation for all the calorimetry results and fits well with the TEM observations. Quantitatively, the enthalpy deviation between heating and cooling gives an estimation of the density of dislocations which have been created during each cycle. For the first cycle, the value of $\Delta H^{h}-\Delta H^{c}$ corresponds to a density of $3 \times 10^{11} \mathrm{~cm}^{-2}$. But this value is certainly overestimated, because energy is also needed for the creation of defects other than dislocations.

\section{v. CONCLUSIONS}

Our calorimetry results show that during thermal cycling through the allotropic transformation of cobalt, the hysteresis increases and the enthalpy change diminishes, results explained by the appearance of transformation-induced defects in the structure, as observed from transmission electron microscopy. The fact that the transformation enthalpy is greater on heating than on cooling is due to the amount of energy needed for their formation.

These transformation-induced defects which remain in the hcp structure are sessile partial dislocations lying in the $\langle 11 \overline{2} 0\rangle$ directions $(\mathbf{b}=\mathbf{p}+\mathbf{c} / 2=1 / 6\langle 2 \overline{2} 03\rangle)$. They can impede the transformation and thus cause an increase of the hysteresis. These dislocations have to be created in the fcc phase in order to adjust each new phase nucleus with the others and with the grain boundaries of the matrix. During each transformation cycle a great part of these dislocations can be pinned and thus they remain in the structure. They are then available for the following transformations, which explains the decrease in the enthalpy change.

\section{REFERENCES}

${ }^{1} \mathrm{~K}$. Shimizu, Proc. of the Int. Symp. on Shape Memory Alloys (China Academic Publishers, 1986), pp. 15-22.

${ }^{2} \mathrm{R}$. Adams and C. Altstetter, Thermodynamics of the Cobalt Transformation, TMS-AIME 242 (1968).

${ }^{3} \mathrm{R}$. Jeanjean, "Influence des Eléments d'Addition sur la Transformation Allotropique du cobalt" (thèse), Service Edition-INSA, $\mathrm{N}^{\circ}$ d'ordre 41, 1972.

${ }^{4}$ A. Seeger, Z. Metallkunde, Bd 47 (1956), Heft 9.

${ }^{5}$ E. M. Kennedy, R. Speiser, and J. P. Hirth, Physical Chemistry in Metallurgy (1976).

${ }^{6}$ O. Blaschko, G. Krexner, J. Pleschiutsching, G. Ernst, C. Hitzenberger, H. P. Karnthaler, and A. Korner, Phys. Rev. Lett. 60, 2800 (1988).

${ }^{7}$ C. Hitzenberger, H. P. Karnthaler, and A. Korner, Acta Metall. 33 (7) (1985)

${ }^{8}$ C. Hitzenberger, H. P. Karnthaler, and A. Korner, phys. stat. sol, (a) 89, 133 (1985).

${ }^{9}$ A. Korner and H. P. Karnthaler, Philos. Mag. A 48 (3) (1983).

${ }^{10}$ H. Bibring, G. Lenoir, and F. Sebilleau, Rev. de métallurgie LVI (3) (1959).

${ }^{11}$ J. L. McNaughton and C.T. Mortimer, "La Calorimétrie Différentielle à Balayage", Perkin-Elmer. 